
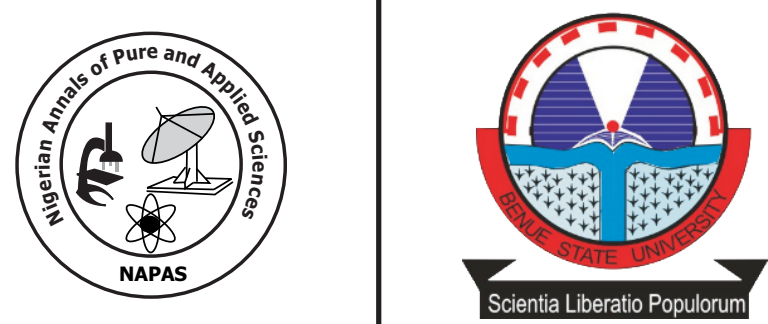

\title{
An Optimized 5-Point Block Formula for Direct Numerical Solution of First Order Stiff Initial Value Problems
}

\author{
${ }^{1}$ Atabo, V. 0. and ${ }^{2}$ Olatunji, P. 0. \\ ${ }^{1}$ Department of Mathematics, Ahmadu Ribadu College, \\ Yola, Adamawa State, Nigeria \\ ${ }^{2}$ Department of Mathematical Sciences, \\ Adekunle Ajasin University, Akungba Akoko, Ondo State, Nigeria, \\ Corresponding Author: atabovictor@gmail.com
}

\begin{abstract}
In this research article, we focus on the formulation of a 5-point block formula for solving first order ordinary differential equations (ODEs). The method is formulated via interpolation and collocation approach using power series expansion as the approximate solution. It has been established that the derived method is of order six. Basic properties such zero and absolute stabilities, convergence, order and error constant have also been investigated. The accuracy of the method was verified on some selected stiff IVPs, compared with some existing methods (DIBBDF, SDIBBDF, BBDF(4), BBDF(5) and odes15s) and test performance showed that the new method is viable.
\end{abstract}

Key words: Absolute stability, stiff initial value problems, block method, error constant. 


\section{Introduction}

The desire to obtain better accurate numerical solu tions to numerous mathematical models, arising from science and engineering and viability of numerical methods to handle the stiffness of the problem has given way for many numerical methods to be developed. Stiffness $y^{\prime}=f(x, y), x \in[a, b], y(a)=\eta$

where, $f$ is continuous and differentiable.

However, $f$ is assumed to satisfy Lipchitz condition and the existence and uniqueness theorem within the interval of $[0,1]$. The system (1) can be regarded as stiff if its exact solution contains very fast and as well as very slow components (Dahlquist, 1974). Nasir, Ibrahim, Suleiman and Othman (2015) considered stable step-size restrictions to some developed block methods and their absolute stabilities. This restriction behaviour to numerical methods makes it difficult to develop suitable methods for solving stiff in itial value problems of ordinary differential equations (ODEs). However, efforts have been made by such researchers asSuleiman, Musa, Ismail, Senu, and Ibrahim(2013), Yap and Ismail (2014, January), Mohamad, Ibrahim and Ismail (2018), Babangida, Musa and Ibrahim (2016) at curbing this restriction via developing methods that are numerically A -stable. Thus, breaking Dahlquist barrier that placed restrictions to step-sizes of numerical methods.

Similarly, various researchers have made giant efforts at tryin $\mathrm{g}$ to develop block methods with higher step length and order. Notable among them are Mohammed and Yahaya (2010), who developed fully implicit four point block method of order four for solving first order ordinary differential equations through interpolation and

$$
y(x)=\sum_{j=0}^{k} a_{j} x^{j}
$$

Taking the first derivative of (2), we get:

$$
y^{\prime}(x)=\sum_{j=0}^{k} j a_{j} x^{j-1}=f(x, y)
$$

remains a difficult and important conc ept in the numerical integration of ODEs. It relies on differential equations, the initial conditions and the interval under consideration (Thohura, 2013). In this research paper, we propose an optimized 5-point block formula for the solution of first order ordinary differential equation of the form:

collocation techniques using power series expansion, Odekunle, Adesanya and Sunday (2012), also formulated 4-point block method of order five for solving first order ordinary differential equations through interpolation and collocation approaches using a combination of power series and exponential function, among others.

It is on the abo ve background, we intend to propose a super block method with higherstep length and order for solving directly stiff IVPs without any provision for starting values, solves more accurately with reduced computational burden than some of the existing numerical methods mentioned above. Derivation of the method is briefly explained in section 2 . In section 3 , the stability properties of the method are discussed. The performances of the method on some stiff problems is presented in comparison to some existing methods in section 4. Section 5 presents discussion of results and a short conclusion is made in the last section.

\section{Derivation of the method}

This section describes the derivation of a 5-point block method which is self -starting for solving (1). For better accuracy of the method in terms of error, we construct the 5 -point block method using power series polynomial as the approximate solution in the form: 
where $a_{j s}$ are parameters to be determined. Thus, we interpolate (2) and collocate (3) at $x_{n+j,} j=0$ and $x_{n+j,} j=0 \ldots 6$ respectively to give the following system of equation using Maple soft environment:

$$
\left(\begin{array}{ccccccc}
1 & x_{n} & x_{n}^{2} & x_{n}^{3} & x_{n}^{4} & x_{n}^{5} & x_{n}^{6} \\
0 & 1 & 2 x_{n} & 3 x_{n}^{2} & 4 x_{n}^{3} & 5 x_{n}^{4} & 6 x_{n}^{5} \\
0 & 1 & 2 x_{n}+2 h & 3\left(x_{n}+h\right)^{2} & 4\left(x_{n}+h\right)^{3} & 5\left(x_{n}+h\right)^{4} & 6\left(x_{n}+h\right)^{5} \\
0 & 1 & 2 x_{n}+4 h & 3\left(x_{n}+2 h\right)^{2} & 4\left(x_{n}+2 h\right)^{3} & 5\left(x_{n}+2 h\right)^{4} & 6\left(x_{n}+2 h\right)^{5} \\
0 & 1 & 2 x_{n}+6 h & 3\left(x_{n}+3 h\right)^{2} & 4\left(x_{n}+3 h\right)^{3} & 5\left(x_{n}+3 h\right)^{4} & 6\left(x_{n}+3 h\right)^{5} \\
0 & 1 & 2 x_{n}+8 h & 3\left(x_{n}+4 h\right)^{2} & 4\left(x_{n}+4 h\right)^{3} & 5\left(x_{n}+4 h\right)^{4} & 6\left(x_{n}+4 h\right)^{5} \\
0 & 1 & 2 x_{n}+10 h & 3\left(x_{n}+5 h\right)^{2} & 4\left(x_{n}+5 h\right)^{3} & 5\left(x_{n}+5 h\right)^{4} & 6\left(x_{n}+5 h\right)^{5}
\end{array}\right)\left(\begin{array}{l}
a_{0} \\
a_{1} \\
a_{2} \\
a_{3} \\
a_{4} \\
a_{5} \\
a_{6}
\end{array}\right)=\left(\begin{array}{c}
f_{n} \\
f_{n+1} \\
f_{n+2} \\
f_{n+3} \\
f_{n+4} \\
f_{n+5} \\
f_{n+6}
\end{array}\right)
$$

Solving for $a_{j s}$ in (4) and then substituted into (2) gives a continuous block implicit method of the form:

$$
y(x)=\sum_{p} \alpha_{p} y_{n+p}+h \sum_{j=0}^{l} \beta_{j} f_{n+j}
$$

where, $\alpha_{j}$ and $\beta_{j}$ are constants; we assume that $\alpha_{k} \neq 0$ and that not both $\alpha_{0}$ and $\beta_{0}$ are zero and that in particular, $p=0$ and $l=5 \mathrm{in}(5)$, so that,

$$
\left.\begin{array}{l}
\alpha_{0}=1 \\
\beta_{0}=t-\frac{137 t^{2}}{120 h}+\frac{5 t^{3}}{8 h^{2}}-\frac{17 t^{4}}{96 h^{3}}+\frac{t^{5}}{40 h^{4}}-\frac{t^{6}}{720 h^{5}} \\
\beta_{1}=\frac{5 t^{2}}{2 h}-\frac{77 t^{3}}{36 h^{2}}+\frac{71 t^{4}}{96 h^{3}}-\frac{7 t^{5}}{60 h^{4}}+\frac{t^{6}}{144 h^{5}} \\
\beta_{2}=-\frac{5 t^{2}}{2 h}+\frac{107 t^{3}}{36 h^{2}}-\frac{59 t^{4}}{48 h^{3}}+\frac{13 t^{5}}{60 h^{4}}-\frac{t^{6}}{72 h^{5}} \\
\beta_{3}=\frac{5 t^{2}}{3 h}-\frac{13 t^{3}}{6 h^{2}}+\frac{49 t^{4}}{48 h^{3}}-\frac{t^{5}}{5 h^{4}}+\frac{t^{6}}{72 h^{5}} \\
\beta_{4}=-\frac{5 t^{2}}{8 h}+\frac{61 t^{3}}{72 h^{2}}-\frac{41 t^{4}}{96 h^{3}}+\frac{11 t^{5}}{120 h^{4}}-\frac{t^{6}}{144 h^{5}} \\
\beta_{5}=\frac{t^{2}}{10 h}-\frac{5 t^{3}}{36 h^{2}}+\frac{7 t^{4}}{96 h^{3}}-\frac{t^{5}}{60 h^{4}}+\frac{t^{6}}{720 h^{5}}
\end{array}\right\}
$$

Evaluating (6) using Maple soft environment at $t=0$ and lgives the following discrete scheme:

$$
y_{n+1}=y_{n}+\frac{95}{288} h f_{n}+\frac{1427}{1440} h f_{n+1}-\frac{133}{240} h f_{n+2}+\frac{241}{720} h f_{n+3}-\frac{173}{1440} h f_{n+4}+\frac{3}{160} h f_{n+5}
$$

Similarly, we obtain the rest of the schemes by solving system of equations to get the $\boldsymbol{\theta}_{j 3}$, interpolating and collocating at $x_{n+j}, 1 j, z, 3,4$ and, $0 \ldots 6 x_{n+j} j=\quad$, that is, taking $p=1,2,3,4$ and $l=5$ in (5) to respectively give:

$$
y_{n+2}=y_{n+1}-\frac{3}{160} h f_{n}+\frac{637}{1440} h f_{n+1}+\frac{511}{720} h f_{n+2}-\frac{43}{240} h f_{n+3}+\frac{77}{1440} h f_{n+4}-\frac{11}{1440} h f_{n+5}
$$




$$
\begin{aligned}
& y_{n+3}=y_{n+2}+\frac{11}{1440} h f_{n}-\frac{31}{480} h f_{n+1}+\frac{401}{720} h f_{n+2}+\frac{401}{720} h f_{n+3}-\frac{31}{480} h f_{n+4}+\frac{11}{1440} h f_{n+5} \\
& y_{n+4}=y_{n+3}-\frac{11}{1440} h f_{n}+\frac{77}{1440} h f_{n+1}-\frac{43}{240} h f_{n+2}+\frac{511}{720} h f_{n+3}+\frac{637}{1440} h f_{n+4}-\frac{3}{160} h f_{n+5} \\
& y_{n+5}=y_{n+4}+\frac{3}{160} h f_{n}-\frac{173}{1440} h f_{n+1}+\frac{241}{720} h f_{n+2}-\frac{133}{240} h f_{n+3}+\frac{1427}{1440} h f_{n+4}+\frac{95}{288} h f_{n+5}
\end{aligned}
$$

Thus, (7)-(11) becomes:

$$
\left.\begin{array}{l}
y_{n+1}=y_{n}+\frac{95}{288} h f_{n}+\frac{1427}{1440} h f_{n+1}-\frac{133}{240} h f_{n+2}+\frac{241}{720} h f_{n+3}-\frac{173}{1440} h f_{n+4}+\frac{3}{160} h f_{n+5} \\
y_{n+3}=y_{n+2}+\frac{11}{1440} h f_{n}-\frac{31}{480} h f_{n+1}+\frac{401}{720} h f_{n+2}+\frac{401}{720} h f_{n+3}-\frac{31}{480} h f_{n+4}+\frac{11}{1440} h f_{n+5} \\
y_{n+4}=y_{n+3}-\frac{11}{1440} h f_{n}+\frac{77}{1440} h f_{n+1}-\frac{43}{240} h f_{n+2}+\frac{511}{720} h f_{n+3}+\frac{637}{1440} h f_{n+4}-\frac{3}{160} h f_{n+5} \\
y_{n+5}=y_{n+4}+\frac{3}{160} h f_{n}-\frac{173}{1440} h f_{n+1}+\frac{241}{720} h f_{n+2}-\frac{133}{240} h f_{n+3}+\frac{1427}{1440} h f_{n+4}+\frac{95}{288} h f_{n+5}
\end{array}\right\}
$$

Hence, ( 12) represents the proposed optimized direct 5-point block formula (D5PBBDF) for the numerical solution of first order ordinary differential equations.

\section{Stability analysis of the method}

Here, we begin the stability analysis of the method by first consider some basic definitions, such as zero stability and A-stability.
Definition 1: A linear multistep method (LMM) is said to be zero stable if no $r$ oot of the first characteristic polynomial has modulus greater than one and that any root with modulus one is simple (that is, not repeated).

Definition 2: A linear multistep method (LMM) is said to be A-stable if its stability region covers the entire negative half-plane.

Equations (12) can be rewritten in matrix form as:

$$
\begin{aligned}
& \left(\begin{array}{lllll}
1 & 0 & 0 & 0 & 0 \\
0 & 1 & 0 & 0 & 0 \\
0 & 0 & 1 & 0 & 0 \\
0 & 0 & 0 & 1 & 0 \\
0 & 0 & 0 & 0 & 1
\end{array}\right)\left(\begin{array}{l}
y_{n+1} \\
y_{n+2} \\
y_{n+3} \\
y_{n+4} \\
y_{n+5}
\end{array}\right)=\left(\begin{array}{lllll}
0 & 0 & 0 & 0 & 1 \\
0 & 0 & 0 & 0 & 1 \\
0 & 0 & 0 & 0 & 1 \\
0 & 0 & 0 & 0 & 1 \\
0 & 0 & 0 & 0 & 1
\end{array}\right)\left(\begin{array}{l}
y_{n-4} \\
y_{n-3} \\
y_{n-2} \\
y_{n-1} \\
y_{n}
\end{array}\right)+\left(\begin{array}{lllll}
0 & 0 & 0 & 0 & 0 \\
1 & 0 & 0 & 0 & 0 \\
0 & 1 & 0 & 0 & 0 \\
0 & 0 & 1 & 0 & 0 \\
0 & 0 & 0 & 1 & 0
\end{array}\right)\left(\begin{array}{l}
y_{n+1} \\
y_{n+2} \\
y_{n+3} \\
y_{n+4} \\
y_{n+5}
\end{array}\right) \\
& +h\left(\begin{array}{cccc}
0000 & \frac{95}{288} \\
0000 & -\frac{3}{160} \\
0000 & \frac{11}{1440} \\
0000 & -\frac{11}{1440} \\
0000 & \frac{3}{160}
\end{array}\right) \\
& +h\left(\begin{array}{llll}
f_{n-4} \\
f_{n-3} \\
f_{n-2} \\
f_{n-1} \\
f_{n}
\end{array}\right) \\
& \left.\begin{array}{ccccc}
\frac{1427}{1440} & -\frac{133}{240} & \frac{241}{720} & -\frac{173}{1440} & \frac{3}{160} \\
\frac{637}{1440} & \frac{511}{720} & -\frac{43}{240} & \frac{77}{1440} & -\frac{11}{1440} \\
-\frac{31}{480} & \frac{401}{720} & \frac{401}{720} & -\frac{31}{480} & \frac{11}{1440} \\
\frac{77}{1440} & -\frac{43}{240} & \frac{511}{720} & \frac{637}{1440} & -\frac{3}{160} \\
-\frac{173}{1440} & \frac{241}{720} & -\frac{133}{240} & \frac{1427}{1440} & \frac{95}{288}
\end{array}\right)\left(\begin{array}{l}
f_{n+1} \\
f_{n+2} \\
f_{n+3} \\
f_{n+4} \\
f_{n+5}
\end{array}\right)
\end{aligned}
$$


Equation (13) can be rewritten as:

$$
A_{0} Y_{m}=A_{1} Y_{m-1}+h\left(B_{0} F_{m-1}+B_{1} F_{m}\right)
$$

where,

$$
\begin{aligned}
& A_{0}=\left(\begin{array}{lllll}
1 & 0 & 0 & 0 & 0 \\
1 & 1 & 0 & 0 & 0 \\
0 & 1 & 1 & 0 & 0 \\
0 & 0 & 1 & 1 & 0 \\
0 & 0 & 0 & 1 & 1
\end{array}\right) ; A_{1}=\left(\begin{array}{ccccc}
0 & 0 & 0 & 0 & 1 \\
0 & 0 & 0 & 0 & 1 \\
0 & 0 & 0 & 0 & 1 \\
0 & 0 & 0 & 0 & 1 \\
0 & 0 & 0 & 0 & 1
\end{array}\right) \\
& B_{0}=\left(\begin{array}{cc}
0000 & \frac{95}{288} \\
0000 & -\frac{3}{160} \\
0000 & \frac{11}{1440} \\
0000 & -\frac{11}{1440} \\
0000 & \frac{3}{160}
\end{array}\right) ; B_{1}=\left(\begin{array}{ccccc}
\frac{1427}{1440} & -\frac{133}{240} & \frac{241}{720} & -\frac{173}{1440} & \frac{3}{160} \\
\frac{637}{1440} & \frac{511}{720} & -\frac{43}{240} & \frac{77}{1440} & -\frac{11}{1440} \\
-\frac{31}{480} & \frac{401}{720} & \frac{401}{720} & -\frac{31}{480} & \frac{11}{1440} \\
\frac{77}{1440} & -\frac{43}{240} & \frac{511}{720} & \frac{637}{1440} & -\frac{3}{160} \\
-\frac{173}{1440} & \frac{241}{720} & -\frac{133}{240} & \frac{1427}{1440} & \frac{95}{288}
\end{array}\right) \\
& Y_{m}=\left(\begin{array}{c}
y_{n+1} \\
y_{n+2} \\
y_{n+3} \\
y_{n+4} \\
y_{n+5}
\end{array}\right) ; Y_{m-1}=\left(\begin{array}{c}
y_{n-4} \\
y_{n-3} \\
y_{n-2} \\
y_{n-1} \\
y_{n}
\end{array}\right) ; F_{m-1}=\left(\begin{array}{c}
f_{n-4} \\
f_{n-3} \\
f_{n-2} \\
f_{n-1} \\
f_{n}
\end{array}\right) ; F_{m}=\left(\begin{array}{c}
f_{n+1} \\
f_{n+2} \\
f_{n+3} \\
f_{n+4} \\
f_{n+5}
\end{array}\right) \\
& Y_{m}=\left(\begin{array}{c}
y_{n+1} \\
y_{n+2}
\end{array}\right) ; Y_{m-1}=\left(\begin{array}{c}
y_{n-1} \\
y_{n}
\end{array}\right) ; F_{m-1}=\left(\begin{array}{c}
f_{n-1} \\
f_{n}
\end{array}\right) ; F_{m}=\left(\begin{array}{c}
f_{n+1} \\
f_{n+2}
\end{array}\right)
\end{aligned}
$$

Substitute the scalar test equation $y^{\prime}=\lambda y$ ( $\lambda<0, \lambda$ is complex) into (14) and taking $\lambda h=\bar{h}$ to get:

$$
A_{0} Y_{m}=A_{1} Y_{m-1}+\bar{h}\left(B_{0} F_{m-1}+B_{1} F_{m}\right)
$$

The method stability polynomial is obtained using Maple soft environment by evaluating:

$$
R(t ; \bar{h})=\operatorname{Det}\left[\left(A_{0}-\bar{h} B_{1}\right) t-\left(A_{1}+\bar{h} B_{0}\right)\right]=0
$$

to give,

$$
\begin{aligned}
& R(t) \bar{h}=-\frac{1}{6} t^{4} \bar{h}^{5}-\frac{79}{18} t^{5} \bar{h}+\frac{77}{12} t^{5} \bar{h}^{2}-\frac{103}{24} t^{5} \bar{h}^{3}+\frac{49}{36} t^{5} \bar{h}^{4}-\frac{1}{6} t^{5} \bar{h}^{5}+t^{5}-t^{4}+\frac{561}{160} t^{4} \bar{h} \\
& -\frac{37937}{12960} t^{4} \bar{h}^{2}+\frac{65}{432} t^{4} \bar{h}^{3}+\frac{221}{360} t^{4} \bar{h}^{4}
\end{aligned}
$$

Zero stabilitv of the method is shown by setting $\bar{h}=0$ in (17) to get:

$R(t ; \bar{h})=t^{5}-t^{4}=0$

Using Maple soft environment to solve (18) gives the following roots:

$t=1, t=0, t=0, t=0, t=0$

Hence, method (12) is zero stable by definition 1.

We present below the absolute stability region of (12) which is determined by taking $t=e^{i \theta}$ into (17). The absolute stability graph is plotted using Matlab soft environment and is given in Figure 1. 


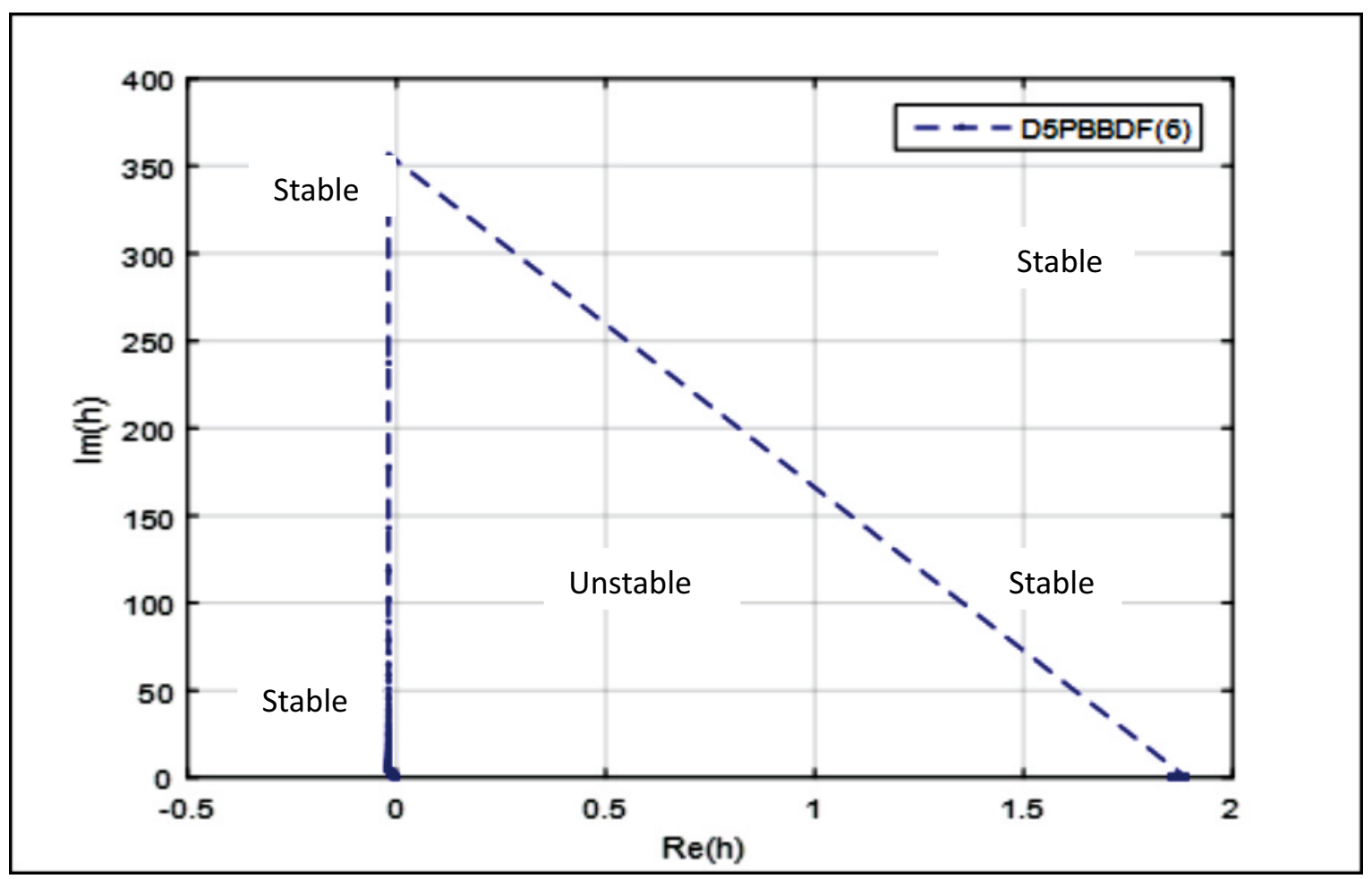

Figure 1: Absolute stability region of $\mathrm{D} 5 \operatorname{PBBDF}(6)$

A clear look at figure 1 indicates that the entire (negative) left half complex plane represents the region of absolute stability of method (12).

\section{Order and error constant}

Maple soft environment investigation of order and error constant in (12) showed that

$$
C_{7}=\left(\begin{array}{c}
-\frac{863}{60480} \\
\frac{271}{60480} \\
-\frac{191}{60480} \\
\frac{271}{60480} \\
-\frac{863}{60480}
\end{array}\right) \text {,implying that it is of order } 6 .
$$

\section{Convergence of the method}

Convergence is a must satisfy property that every linear multistep method (LMM) must have. We shall discuss convergence of method (12), which entails that it must be zero-stable and consistent. However, (12) has already been established to be zero-stable above.

$\sum_{j=0}^{3} D_{j}=0$,

$\sum_{j=0}^{3} j D_{j}=\sum_{j=0}^{3} G_{j}$

where, $D_{j^{\prime} s}$ and $\mathrm{G}_{j^{\prime} s}$ are matrices.

Definition 3 : Method (12) is co nsistent if and only if the following conditions are fulfilled: The order $p \geq 1$ 


\section{Remark:}

Condition (19) is sufficient for the associated block method to be consistent, i.e. $p \geq 1$ Jator (2007).

Thus, (12) is consistent since the order $p=6>1$. Since, the method is both zero stable and consistent, it thus converges.

\section{Implementation of the method}

Since the new method is in itself self starting, its implementation is direct and as such, does not require using a predictor formula. Hence, all approximate solutions are obtained simultaneously in block using Maple soft environment (MAPLE 18).

\section{Definition 4: Let $y_{i}$ and $y\left(x_{i}\right)$ be the} approximate and exact solution of (1) respectively, then the maximum error is evaluated by using the formula:

$\operatorname{MAXE}=\max _{\mathbb{E} \leq \leq N S}\left|\left(y_{i}\right)_{t}-\left(y\left(x_{i}\right)\right)_{t}\right|$

where, NS is the total number of steps.

\section{Test Examples}

The following first order stiff initial value problems in (ODEs) are used to prove the accuracy of the method.

Example 1: [Nasir, et al.,(2015)]

$y^{\prime}=-1000 y+3000-2000 e^{-x}, y(0)=0,0 \leq x \leq 1$

Exact solution: $y(x)=3-0.998 e^{-1000 x}-2.002 e^{-x}$

Eigenvalue $\lambda=-1000$
Example 2: [Nasir, et al.,(2015)]

$y^{\prime}=-1000 y+3000-2000 e^{-x}, y(0)=0,0 \leq x \leq 1$

Exact solution: $y(x)=3-0.998 e^{-1000 x}-2.002 e^{-x}$

Eigenvalue: $\lambda=-1000$

Example 3: [Aksah, et al., (2019)]

$y^{\prime}=-100(y-\sin x)+\cos x, y(0)=0,0 \leq x \leq 1$

Exact solution: $y(x)=\sin x$

Eigenvalue: $\lambda=-100$

\section{Numerical results}

The tables below show the results from applying the new method (12) with comparison to some existing numerical methods in terms of absolute maximum error. The following notations interpret the elements in the tables:

SDIBBDF : Singly diagonally implicit BBDF method by Aksah et al., (2019)

BBDF(4) and BBDF(5) : Block Method for Multistep Formulas for Solving Ordinary Differential Equations, by Ibrahim (2006).

DIBBDF: Diagonally implicit BBDF formulas for solving fuzzy differential equations by Zawawi, (2014) odes $15 \mathrm{~s}$ : VSVO solver based on the numerical differentiation formulas (NDFs)

NS : Number of steps taken

$h:$ Stepsize

MAXE : Maximum error

Table 1: Numerical results for example 1

\begin{tabular}{clcc}
\hline Step-size (h) & Method & NS & MAXE \\
\hline \multirow{2}{*}{$\mathbf{1 0}^{-\mathbf{3}}$} & BBDF(4) & 1000 & $7.27898 \mathrm{e}+108$ \\
& BBDF(5) & 1000 & $8.55887 \mathrm{e}+202$ \\
& D5PBBDF(6) & 1000 & $7.33700 \mathrm{e}-007$ \\
& & 10000 & \\
& BBDF(4) & 10000 & $1.43379 \mathrm{e}-001$ \\
\multirow{2}{*}{$\mathbf{1 0}^{-4}$} & BBDF(5) & 10000 & $7.65939 \mathrm{e}-003$ \\
& D5PBBDF(6) & & $7.30700 \mathrm{e}-007$ \\
\hline
\end{tabular}


Table 2: Numerical results for Example 2

\begin{tabular}{|c|c|c|c|}
\hline \multirow[t]{2}{*}{ Step-size (h) } & \multirow[t]{2}{*}{ Method } & \multicolumn{2}{|r|}{ MAXE } \\
\hline & & NS & \\
\hline \multirow{4}{*}{$10^{-2}$} & $\operatorname{BBDF}(4)$ & & $8.28814 \mathrm{e}+006$ \\
\hline & $\operatorname{BBDF}(5)$ & 100 & $5.18981 \mathrm{e}+013$ \\
\hline & D5PBBDF(6) & & $1.10000 \mathrm{e}-009$ \\
\hline & & 100 & \\
\hline \multirow{6}{*}{$10^{-3}$} & & 100 & \\
\hline & $\operatorname{BBDF}(4)$ & & $1.55450 \mathrm{e}-003$ \\
\hline & $\operatorname{BBDF}(5)$ & 1000 & $1.67200 \mathrm{e}-005$ \\
\hline & D5PBBDF(6) & & $2.60000 \mathrm{e}-009$ \\
\hline & & 1000 & \\
\hline & & 1000 & \\
\hline
\end{tabular}

Table 3: Numerical results for Example 3

\begin{tabular}{llll}
\hline Step-size (h) & Method & NS & MAXE \\
\hline \multirow{2}{*}{$\mathbf{1 0}^{-\mathbf{2}}$} & DIBBDF & - & $9.11949 \mathrm{e}+000$ \\
& SDIBBDF & - & $5.50135 \mathrm{e}-003$ \\
& Ode15s & - & $1.21111 \mathrm{e}-004$ \\
& D5PBBDF(6) & 300 & $1.27000 \mathrm{e}-010$ \\
& & & \\
\multirow{2}{*}{$\mathbf{1 0}^{-4}$} & DIBBDF & - & $7.14971 \mathrm{e}-005$ \\
& SDIBBDF & - & $1.20673 \mathrm{e}-006$ \\
& Ode15s & - & $3.20516 \mathrm{e}-006$ \\
& D5PBBDF(6) & & $6.27000 \mathrm{e}-010$ \\
\hline
\end{tabular}

\section{Discussion}

A vivid look at Table 1 shows that with step-size $h=10^{-3} \operatorname{BBDF}(4)$ has its absolute maximum error as $7.27898 e+108, \operatorname{BBDF}(5)$ has $8.55887 e+202$ while the new method D5PBBDF(6) $7.33700 e-007$ and for step -size $h=10^{-4}, \quad \operatorname{BBDF}(4)$ has $1.43379 e-001$, $\operatorname{BBDF}(5)$ has $4.65939 e-003$ while the new method D5BBDF(6) has 7.30700 e-007. Also, Table 2 shows that BBDF(4) has 8.28814 $e+006$ , $\operatorname{BBDF}(5)$ has $5.18981 e+013$ while the new method D5BBDF(6) has $1.10000 e-009$. Also, with step -size $h=10^{-3}, \operatorname{BBDF}(4)$ has absolute maximum error of $1.55450 e-003, \operatorname{BBDF}(5)$ has $1.67200 e-005$ while D5PBBDF(6) has $2.60000 e-009$.

Table 3 also indicates that with a step size, $h=10^{-2}$ for problem 3 , the existing methods DIBBDF has absolute maximum error of

9.11949e+000, SDIBBDF has 5.50135e-003, Ode15s has $1.21111 e-004$ while the $\mathrm{n}$ ew method D5BBDF(6) has $1.27000 \quad e-010$, implying that the new method has small scale error than the compared methods respectively.

Similarly, with $h=10^{-4}$, DIBBDF has absolute maximum error of $7.14971 e-005$, SDIB BDF has $1.20673 e-006$, Ode15s has $3.20516 e-006$ while the new method D5BBDF(6) has $6.27000 e-010$ indicating that the new method D5BBDF(6) has better accuracy than some of the existing methods DIBBDF, SDIBBDF, and ode $15 \mathrm{~s}$ considered in terms of absolute maximum error respectively. Evidently, convergence of the new method indicated that the new method has some level of consistency in terms of maximum errors of all the test problems considered. Thus, a unique feature of the developed formula. However, the maximumerror also implied that the approximate solutions tend to the exact solution as the iteration processes continue. Hence, the new method converges faster than the existing methods on the respective problems considered. Below are the efficiency curves comparing all methods considered with respect to the individual stiff IVPs. The curves are plotted using Matlab soft environment. 


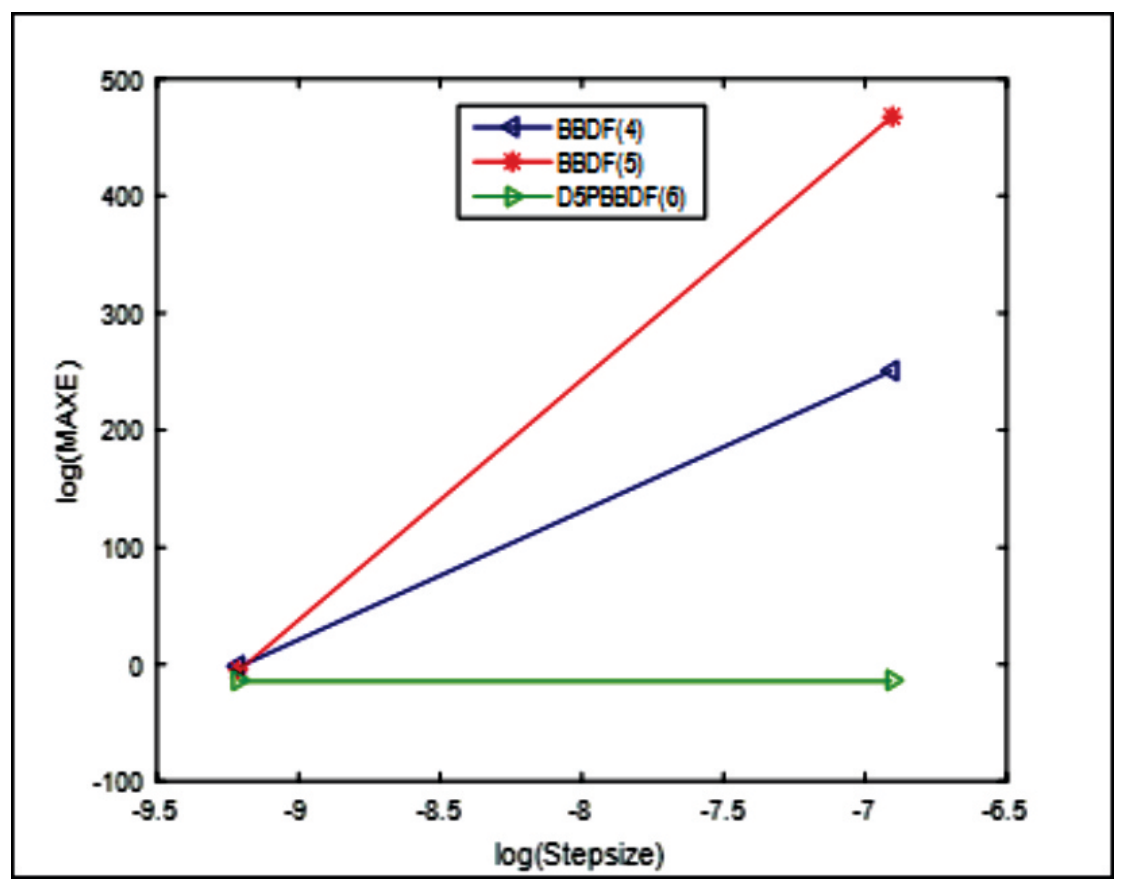

Figure 2: Comparison of efficiency curves in terms of error for Example 1

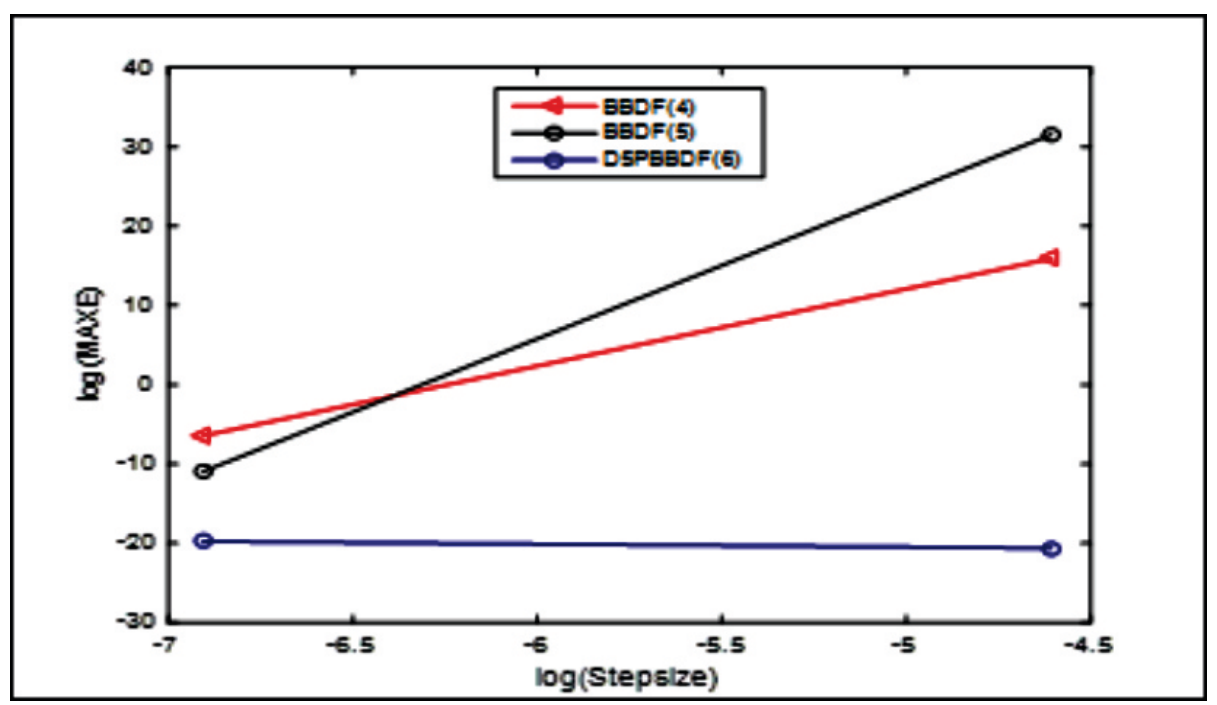

Figure 3: Comparison of efficiency curves in terms of error for problem 2

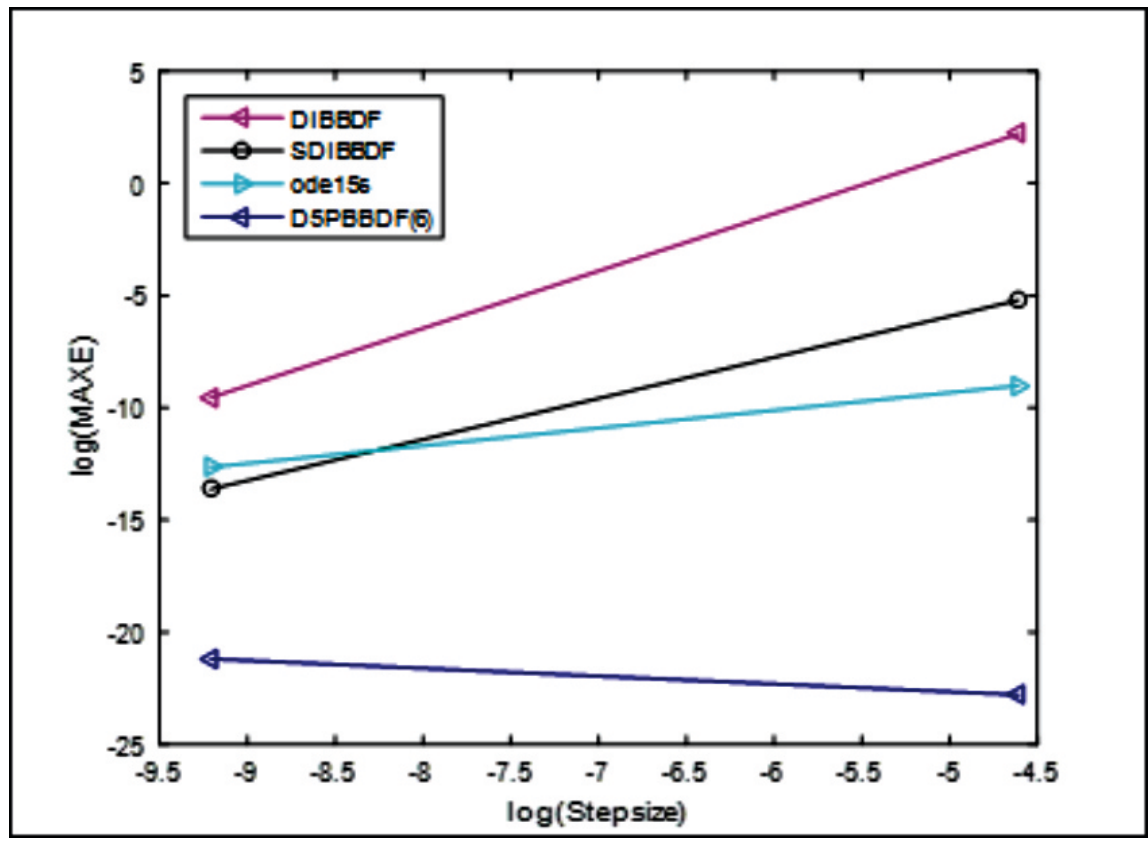

Figure 4: Comparison of efficiency curves in terms of error for problem 3 
The efficiency curves in figure 2-4 indicate clearly the viability of the new method over some of the compared existing methods. Numerically, the graphs implied that the scaled error of the new method is smaller when compared with the existing methods under consideration.

\section{Conclusion}

A new method an optimized 5-point BBDF has been developed through interpolation and collocation using power series expansion as the approximate solution. The method is of a higher block and order. Using Maple soft environment, it has been established that the schemes which form the 5-point block are of a uniform order six. Region of absolute stability showed that the method is A-stable. It also showed that it has a smaller unstable region. The efficiency of the method on test examples showed that it has better level of accuracy than some compared existing methods such as BBDF(4), BBDF(5), DIBBDF, SDIBBDF and ode15 respectively. Hence, a new method for solving first order stiff initial value problems in ordinary differential equations (ODEs) has been formulated.

\section{References}

Aksah, J. S., Ibrahim, Z. B. and Zawawi, I. S. M. (2019). Stability Analysis of Singly Diagonally I m p li c i t B lo c k Backward Differentiation Formulas for Stiff Ordinary Differential Equations. Research gate Publication. http://doi.org/10.3390/math7020211.

Babangida, B., Musa, H. \& Ibrahim, L. K. (2016). A New Numerical Method for solving Stiff Initial value Problems. Fluid Mechanics: Open Access. 3(2), 1-5. http://doi.org/ 10.4172/2476-2296.1000136.

Dahlquist, G. (1974). Problems related to the numerical treatment of stiff differential equations. International computing symposium. North Holland, Amsterdam, pp. 307-314.

Ibrahim, Z. B. (2006). Block Method for Multistep Formulas for Solving Ordinary Differential Equations. Ph.D. Thesis, Universiti Putra, Malaysia,
Selangor, Malaysia.

Jator, S. N. (2007). A sixth order linear multistep method for the direct solution of second order ordinary differential equations. International Journal of Pure and Applied Mathematics. 40(4), 457-472.

Mohamad, N. N., Ibrahim, Z. B. \& Ismail, F. (2018). Numerical Solution for Stiff Initial Value Problems Using 2-point Block Multistep Method. Vol. 1132, IOP Publishing Ltd.

Mohammed, U. and Yahaya, Y. A. (2010). Fully implicit four point block backward difference formula for solving firstorder initial value problems. Leonardo Journal of Sciences, 16(1), 21-30.

Nasir, N. A. M., Ibrahim, Z. B., Suleiman, M., and Othman, K. I. (2015). Stability of block backward differentiation formulas method. AIP Conference Proceedings, 1682, 020010 ; https://doi.org/10.1063/1.4932419

.Odekunle, M. R., Adesanya, A. O. and Sunday, J. (2012). 4-Point Block Method for Direct Integration of First-Order Ordinary Differential Equations. Internal Journal of Engineering Research and Applications (IJERA), 2(5), 1182-1187.

Suleiman, M. B., Musa, H., Ismail, F., Senu, N. \& Ibrahim, Z. B. (2013). An accurate block solver for stiff IVPs. ISRN, Applied Mathematics.

Thohura, S. \& Rahma, A. (2013). Numerical Approach for Solving Stiff Differential Equation: A Comparative study. Global Journal of Science Frontier Research Mathematics and Decision Science, 13(6), 1-13.

Yap, L. K., and Ismail, F. (2014, January). Block methods with off-steps points for solving first order ordinary differential equations. In International Conference on Mathematical Sciences and Statistics, (2013), 275-284. Springer Singapore.

Zawawi, I. S. M. (2014). Diagonally Implicit Block Backward Differentiation Formulas for Solving Fuzzy Differential Equations. Master's Thesis, Universiti Putra Malaysia, Selangor, Malaysia. 